\title{
The cardiovascular risks of NSAID use: which NSAID is the safest of them all?
}

$\mathrm{N}$ SAIDs are the principal agents used for managing pain in patients with chronically painful diseases, such as osteoarthritis. However, the withdrawal of the selective cyclooxygenase 2 (COX2) inhibitor rofecoxib in 2004, based on evidence of an increased risk of adverse cardiovascular events in users of this drug, has led to debate about the safety of other selective COX2 inhibitors and traditional NSAIDs. According to the available data, the safety profiles of individual NSAIDs vary markedly, leading clinicians to ask which one has the best cardiovascular safety. A team of investigators based in Switzerland have attempted to answer this question by means of a network meta-analysis, the results of which have now been published in the British Medical Journal.

According to Peter Jüni, the corresponding author of the paper, previous efforts at a comprehensive comparison of the cardiovascular safety of NSAIDs have not passed muster. "Several conventional meta-analyses were unable to resolve the debate, as they did not integrate all the randomized evidence available into one single analysis," he explains, "which is exactly what network meta-analysis does: it allows a unified, coherent analysis of all randomized controlled trials that compare NSAIDseither head-to-head or with placebowhile still respecting randomization."

The analysis included a total of 31 randomized controlled trials, incorporating data from 116,429 patients with 115,000 patient-years of follow-up. Small studies (defined as those with $<100$ patient-years of follow-up) were excluded. All the included trials compared any NSAID with another NSAID, paracetamol or placebo in patients with any medical condition. Seven NSAIDs were compared in the analysis: four selective COX2 inhibitors (celecoxib, etoricoxib, rofecoxib and lumiracoxib)

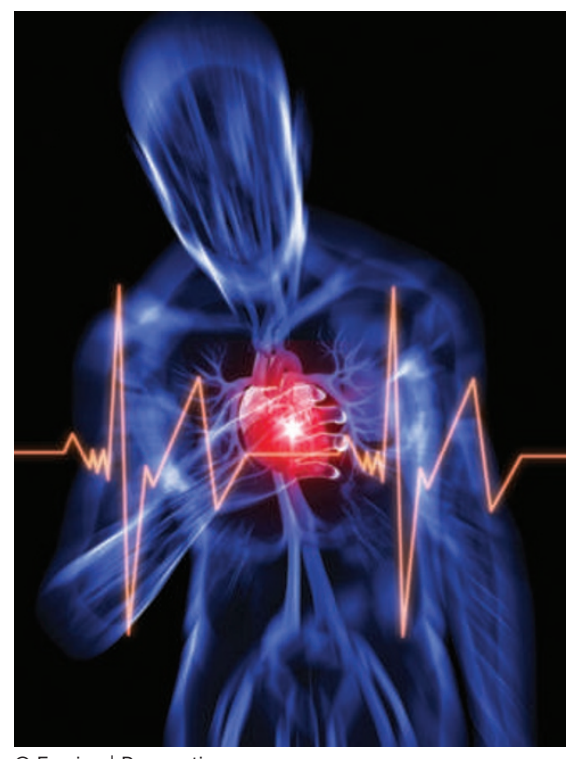

() Eraxion | Dreamstime

and three traditional NSAIDs (naproxen, ibuprofen and diclofenac).

The primary outcome measure was myocardial infarction (fatal or nonfatal). Secondary outcome measures were stroke (fatal or nonfatal), cardiovascular death (death attributed to any cardiovascular cause or an unknown cause), death by any cause, and the Antiplatelet Trialists' Collaboration (APTC) composite outcome (nonfatal myocardial infarction, nonfatal stroke or cardiovascular death). The risk of each of these outcomes was estimated as a rate ratio (RR) for each NSAID compared with placebo. To take into account the imprecise nature of the estimates generated from the available data, which included generally low numbers of recorded cardiovascular events, an RR of 1.3 was prespecified as the threshold for a 'clinically significant' increased risk.

"We found several clinically relevant toxicity signals, and the individual safety profiles of the drugs varied markedly depending on the outcome measure used," says Jüni. "In particular, we found that cardiovascular risk is not clearly associated with NSAIDs that display selective COX2 inhibition. This suggests that cardiovascular risk cannot be predicted on the basis of such specificity."

Compared with placebo, rofecoxib was associated with the highest risk of myocardial infarction (RR 2.12), closely followed by lumiracoxib (RR 2.00), with naproxen, diclofenac and etoricoxib registering the lowest risks ( $R R 0.82$, 0.82 and 0.75 , respectively). However, etoricoxib and diclofenac were associated with the highest risks of cardiovascular death (RR 4.07 and 3.98, respectively), with naproxen again showing a low risk when compared with placebo (RR 0.98). Indeed, by most of the measures analyzed, naproxen seemed to demonstrate the most favorable cardiovascular safety profile of all the NSAIDs included. According to Jüni, however, "this advantage has to be considered against naproxen's gastrointestinal toxicity profile, and the need for concomitant proton pump inhibitor use in many patients."

Ultimately, this network meta-analysis does not seem able to provide the ultimate, definitive answer to the question of which NSAID is the safest for treating patients with chronic musculoskeletal pain-a group of patients who are, for a number of reasons, often already at increased risk of cardiovascular events. The alternatives to NSAIDs in these patients are few, and all have their own limitations in terms of efficacy and safety. Thus, it seems that cardiovascular risk must continue to be a key consideration when prescribing NSAIDs for chronic pain, and close monitoring of patients receiving long-term NSAID therapy remains essential.

Nick Warde 